\title{
TOP2A, GGH, and PECAM1 are associated with hematogenous, lymph node, and peritoneal recurrence in stage II/III gastric cancer patients enrolled in the ACTS-GC study
}

\author{
Masanori Terashima ${ }^{1, *}$, Wataru Ichikawa ${ }^{2, *}$, Atsushi Ochiai ${ }^{3}, K_{0 j i}$ Kitada $^{4}$, Issei \\ Kurahashi ${ }^{5}$, Shinichi Sakuramoto ${ }^{6}$, Hitoshi Katai ${ }^{7}$, Takeshi Sano $^{8}$, Hiroshi Imamura9 , \\ and Mitsuru Sasako ${ }^{10}$; for the ACTS-GC Group \\ ${ }^{1}$ Division of Gastric Surgery, Shizuoka Cancer Center, Shimonagakubo, Nagaizumi-cho, Sunto-gun, Shizuoka, Japan \\ ${ }^{2}$ Division of Medical Oncology, Showa University Fujigaoka Hospital, Kanagawa, Japan \\ ${ }^{3}$ Division of Pathology, National Cancer Center Hospital East, Kashiwanoha, Kashiwa, Chiba, Japan \\ ${ }^{4}$ Department of Surgery, National Hospital Organization Fukuyama Medical Center, Okinogami-cho, Fukuyama, Hiroshima, \\ Japan \\ ${ }^{5}$ Data Innovation Center, iAnalysis LLC, Minamiaoyama, Minato-ku, Tokyo, Japan \\ ${ }^{6}$ Department of Surgery, Saitama Medical University International Medical Center, Yamane, Hidaka, Saitama, Japan \\ ${ }^{7}$ Gastric Surgery Division, National Cancer Center Hospital, Tsukiji, Chuo, Tokyo, Japan \\ ${ }^{8}$ Department of Gastroenterological Surgery, Cancer Institute Hospital, Japanese Foundation for Cancer Research, Ariake, \\ Koto-Ku, Tokyo, Japan \\ ${ }^{9}$ Department of Surgery, Toyonaka Municipal Hospital, Shibahara-cho, Toyonaka, Osaka, Japan \\ ${ }^{10}$ Department of Surgery, Hyogo College of Medicine, Mukogawa-cho, Nishinomiya, Hyogo, Japan \\ * These authors have contributed equally to this work \\ Correspondence to: Masanori Terashima, email: m.terashima@scchr.jp \\ Keywords: stomach neoplasm, DNA topoisomerase II alpha, gamma-glutamyl hydrolase, CD31 \\ Received: September 24, 2016 Accepted: February 12, $2017 \quad$ Published: March 04, 2017
}

Copyright: Terashima et al. This is an open-access article distributed under the terms of the Creative Commons Attribution License 3.0 (CC BY 3.0), which permits unrestricted use, distribution, and reproduction in any medium, provided the original author and source are credited.

\section{ABSTRACT}

Background: To identify factors related to relapse sites, we carried out an exploratory biomarker analysis of data from the Adjuvant Chemotherapy Trial of TS-1 for Gastric Cancer study, which is a randomized, controlled trial comparing postoperative adjuvant S-1 therapy with surgery alone in 1,059 patients with stage II/III gastric cancer.

Patients and Methods: Surgical specimens from 829 patients were retrospectively examined, and 63 genes involved in a variety of biological processes were analyzed by quantitative real-time PCR. Gene expression normalized to reference genes was categorized as lower or higher than the median, and association with relapse sites was analyzed based on 5-year relapse-free survival.

Results: Hematogenous, lymph node, and peritoneal recurrence developed in 72,105 , and 138 of the 829 patients, respectively; hazard ratios were 0.79 (95\% confidential interval: 0.54-1.16), $0.51(0.31-0.82)$, and $0.60(0.42-0.84)$, respectively. Expression of platelet/endothelial cell adhesion molecule 1 (PECAM1) and topoisomerase II alpha (TOP2A) was strongly correlated with hematogenous recurrence and peritoneal recurrence, respectively (false discovery rate $=7.7 \times 10^{-5}$ and 0.002 , respectively). Gamma-glutamyl hydrolase (GGH) expression was moderately correlated with lymph node recurrence (false discovery rate $=\mathbf{0 . 3 4}$ ). Relapse-free survival was worse in patients expressing high levels of PECAM1 (hazard ratio $=2.37$, 1.65-3.41), TOP2A (hazard ratio $=2.35,1.55-3.57$ ), or GGH (hazard ratio $=1.87$, 


\subsection{3-3.08), respectively. A multivariate analysis revealed that these were stronger independent risk factors than tumor histological type. \\ Conclusion: In patients with stage II/III gastric cancer, TOP2A, GGH, and PECAM1 levels in primary tumors are linked to high risk of hematogenous, lymph node, and peritoneal recurrence, respectively.}

\section{INTRODUCTION}

Despite a decreasing trend, gastric cancer remains the most common malignancy of the gastrointestinal tract in Japan and the second most common cause of cancer-related death worldwide. Gastrectomy with D2 lymphadenectomy on stage II or III gastric cancer followed by adjuvant chemotherapy with TS-1 - an orally active combination of tegafur, gimeracil, and oteracil—has shown a favorable outcome. The Adjuvant Chemotherapy Trial of TS-1 for Gastric Cancer (ACTS-GC) - a prospective, randomized phase III trial of Japanese patients with stage II/III gastric cancer-revealed a 5-year survival rate of $50.2 \%-84.2 \%$ [1-3]. Nonetheless, some cancers recur after surgery in the form of blood-borne metastasis or peritoneal dissemination; moreover, the high variability among recurrence patterns impedes proper treatment. Hence, understanding the factors that influence recurrence can lead to the development of more effective treatments or optimization of surveillance procedures for patients with high-risk gastric cancer who undergo curative resection.

Tumor histology is correlated with the metastatic behavior of cancer cells. According to Lauren's classification, diffuse-type gastric cancer is characterized by infiltration of neoplastic cells with a significant desmoplastic response and early spreading via lymphatic dissemination, local extension into neighboring organs, or as peritoneal carcinomatosis [4]. Hepatic metastases are frequently observed in intestinal-type gastric cancercharacterized by cohesive carcinoma cells forming glandlike tubular structures with an expanding or infiltrative growth pattern - due to drainage into the portal vein. Gene expression profiles associated with cancer metastasis have been extensively studied using genome-wide approaches such as DNA microarray [5-9] and have been used to predict peritoneal relapse after curative surgery for gastric cancer [10]. An eight-gene Gastric Cancer Prognostic Score has been developed to identify patients with stage II gastric cancer who are at high risk of recurrence after surgery regardless of adjuvant treatment [11]. However, predicting metastatic risk based solely on tissue type is inadequate; furthermore, it is necessary to analyze other cohorts since previous gene profiling-based studies have been exploratory. Identifying specific genes whose expression is correlated with gastric cancer metastasis would provide biomarkers that can be used to assess the risk of recurrence.

The present study investigated gene expression in primary gastric cancer tissue in order to identify patients at a high risk for relapse at specific sites. Gene expression levels in gastric tumor tissue obtained from patients enrolled in the ACTS-GC study were evaluated by quantitative real-time PCR. In addition, we examined the relationship between the expression level of each gene and patient outcome, especially in relation to tumor relapse sites.

\section{RESULTS}

\section{Demographics of the study population}

Archived FFPE specimens obtained by surgical resection were available for $829(78.3 \%)$ of the 1,059 patients enrolled in the ACTS-GC trial at 65 centers who constituted the biomarker study population. Patient demographic data and tumor characteristics (Supplementary Table 1) are summarized elsewhere [12]. The median patient age was 62 years (range: $27-80$ years). As previously reported, there was no significant difference between the patients included in the current biomarker study and the total ACTS-GC trial study population.

\section{Gene expression}

Gene expression stability measures for the reference genes $\beta$-actin, glyceraldehyde 3-phosphate dehydrogenase, and ribosomal protein lateral stalk subunit $P 0$ were calculated as $0.916,0.931$, and 0.923 , respectively. The $\mathrm{M}$ values were $<1.5$ for all three genes, indicating that they were suitable for target gene normalization. Four genes (cyclin-dependent kinase inhibitor 2A, epidermal growth factor, insulin-like growth factor 2, and semaphorin $3 B$ ) were excluded from further analyses because their expression levels were below the detection limit in $<60 \%$ of the samples $(51.5 \%, 23.6 \%$, $44.5 \%$, and $5.5 \%$, respectively). Thus, 59 of the 63 genes subjected to LDA met quality control criteria. The median success rate for the 59 genes was $98.6 \%$ (range: $61.2 \%$ $100 \%)$.

\section{Site of first relapse}

During the 5-year follow-up period, 291/829 patients (35.1\%) experienced gastric cancer recurrence; the peritoneum, hematogenous sites, and lymph nodes were the most common sites of first relapse. Rates of metastasis 
Table 1: Site of first relapse according to treatment group*

\begin{tabular}{|l|l|l|l|l|}
\hline Site & $\begin{array}{l}\text { S-1 } \\
(\boldsymbol{n}=\mathbf{4 1 5})\end{array}$ & $\begin{array}{l}\text { Surgery-only } \\
(\boldsymbol{n}=\mathbf{4 1 4})\end{array}$ & HR & $\mathbf{9 5 \%}$ CI \\
\hline No. of relapses & $123(29.6 \%)$ & $175(42.3 \%)$ & & \\
\hline Local & $9(2.2 \%)$ & $14(3.4 \%)$ & 0.557 & $0.241-1.287$ \\
Lymph nodes & $26(6.3 \%)$ & $46(11.1 \%)$ & 0.507 & $0.313-0.820$ \\
Peritoneum & $56(13.5 \%)$ & $83(20.0 \%)$ & 0.595 & $0.424-0.836$ \\
Hematogenous & $49(11.8 \%)$ & $56(13.5 \%)$ & 0.787 & $0.538-1.155$ \\
\hline
\end{tabular}

* Some patients experienced first relapse at more than one site.

$\mathrm{CI}$, confidence interval; $\mathrm{HR}$, hazard ratio

and relapse were lower in the S-1 than in the surgery-only group for all sites (Table 1 and Figs. S1 and S2). Two hundred forty-seven patients $(29.8 \%)$ had recurrence at a single site, 40 patients $(4.8 \%)$ had recurrence at two sites, and four patients $(0.5 \%)$ had recurrence at three site. (Fig. 1).

\section{Risk factors for recurrence at each site}

We analyzed the association between gene expression level and each recurrence site with the proportional hazard model (Table 2 and Figure 2). Among the 56 screened genes, gamma-glutamyl hydrolase $(G G H)$ was most highly correlated with lymph node recurrence $(\mathrm{FDR}=0.354)$. Lymph node relapse-free survival was lower in patients with high as compared to low $G G H$ expression [hazard ratio $(\mathrm{HR})=1.87 ; 95 \%$ confidence interval (CI): 1.13-3.08]. The cumulative recurrence rate was higher in patients with high as compared to low GGH levels. Platelet/endothelial cell adhesion molecule $(P E C A M) 1$ was most highly correlated with recurrence in the peritoneum [False discovery rate $\left.(\mathrm{FDR})=7.7 \times 10^{-5}\right]$. Cumulative peritoneal recurrence rate was higher in patient with high as compared to low PECAM1 expression $(\mathrm{HR}=2.37 ; 95 \% \mathrm{CI}: 1.65-3.41)$. Topoisomerase II alpha (TOP2A) was most highly correlated with hematogenous recurrence $(\mathrm{FDR}=0.002)$; the cumulative hematogenous recurrence rate was higher in patients with high as compared to low TOP $2 A$ levels $(\mathrm{HR}=2.35$; 95\% CI: 1.55 3.57). There were no statistically significant interactions between $G G H, P E C A M 1$, or TOP2A expression and $\mathrm{S}-1$ treatment at the respective recurrence sites, and no statistically significant factors were correlated with local recurrence (Supplementary Table 2).

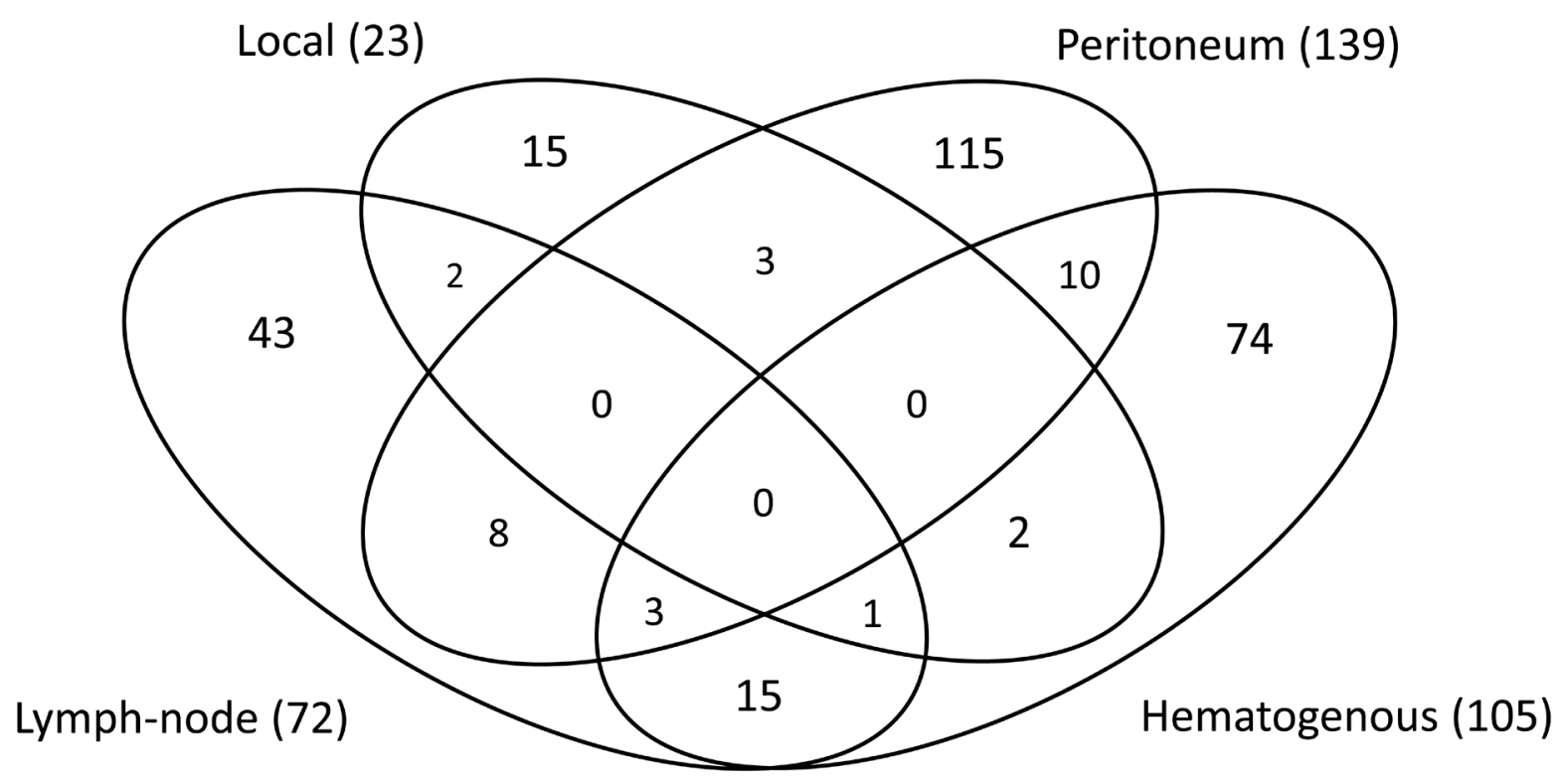

Figure 1: Recurrence patterns in 298 patients with documented relapse after complete resection of gastric adenocarcinoma. 
Table 2: Risk factors for recurrence at each site

\begin{tabular}{|c|c|c|c|c|c|c|c|c|c|c|c|c|c|c|}
\hline \multicolumn{5}{|c|}{ Lymph-node recurrences } & \multicolumn{5}{|c|}{ Peritoneum recurrence } & \multicolumn{5}{|c|}{ Hematogenous recurrence } \\
\hline Gene & H.R. & $95 \% \mathrm{CI}$ & $\begin{array}{l}\text { Log- } \\
\text { Rank P }\end{array}$ & FDR & Gene & H.R. & $95 \% \mathrm{CI}$ & $\begin{array}{l}\text { Log- } \\
\text { Rank P }\end{array}$ & FDR & Gene & H.R. & $95 \% \mathrm{CI}$ & $\begin{array}{l}\text { Log- } \\
\text { Rank P }\end{array}$ & FDR \\
\hline$G G H$ & 1.869 & $1.134-3.080$ & 0.013 & 0.354 & PECAMI & 2.373 & $1.652-3.408$ & $1.38 \mathrm{E}-06$ & $7.72 \mathrm{E}-05$ & TOP $2 A$ & 2.353 & $1.552-3.568$ & $3.3 \mathrm{E}-05$ & $\begin{array}{l}1.8 \mathrm{E}- \\
03\end{array}$ \\
\hline$A B C B I$ & 0.546 & $0.328-0.908$ & 0.018 & 0.354 & $A B C C l$ & 2.147 & $1.498-3.077$ & 0.00002 & 0.00056 & $E R B B 2$ & 2.227 & $1.484-3.342$ & 7.2E-05 & $\begin{array}{l}2.0 \mathrm{E}- \\
03\end{array}$ \\
\hline UPPI & 0.569 & $0.353-0.917$ & 0.019 & 0.354 & THBSI & 2.052 & $1.444-2.917$ & 4.2E-05 & $7.8 \mathrm{E}-04$ & $G Z M A$ & 0.445 & $0.286-0.693$ & 0.000 & 0.004 \\
\hline ESRI & 0.580 & $0.353-0.954$ & 0.030 & 0.377 & TOP $2 A$ & 0.489 & $0.342-0.699$ & $6.0 \mathrm{E}-05$ & $8.5 \mathrm{E}-04$ & $G G H$ & 2.155 & $1.413-3.287$ & 0.000 & 0.004 \\
\hline$E R B B 2$ & 1.657 & $1.035-2.655$ & 0.034 & 0.377 & $C A V I$ & 1.998 & $1.406-2.840$ & $8.2 \mathrm{E}-05$ & $9.2 \mathrm{E}-04$ & $F A S$ & 0.486 & $0.318-0.744$ & 0.001 & 0.008 \\
\hline TYMP & 0.651 & $0.406-1.045$ & 0.073 & 0.637 & $G G H$ & 0.489 & $0.335-0.715$ & 0.000 & 0.001 & ESRI & 0.512 & $0.337-0.776$ & 0.001 & 0.012 \\
\hline$B A X$ & 0.674 & $0.421-1.080$ & 0.099 & 0.637 & $I G F I R$ & 1.916 & $1.356-2.708$ & 0.000 & 0.001 & $L R P 5$ & 1.880 & $1.262-2.803$ & 0.002 & 0.013 \\
\hline TOP $2 A$ & 1.467 & $0.918-2.343$ & 0.107 & 0.637 & $I T G B 3$ & 1.901 & $1.336-2.705$ & 0.000 & 0.002 & $V E G F A$ & 1.745 & $1.175-2.593$ & 0.005 & 0.037 \\
\hline PECAMI & 0.692 & $0.432-1.108$ & 0.123 & 0.637 & $E Z H 2$ & 0.518 & $0.360-0.745$ & 0.000 & 0.002 & PTGS2 & 0.635 & $0.426-0.948$ & 0.025 & 0.144 \\
\hline$F A S$ & 0.691 & $0.425-1.122$ & 0.133 & 0.637 & $T Y M S$ & 0.564 & $0.399-0.798$ & 0.001 & 0.006 & $E G F R$ & 1.571 & $1.053-2.344$ & 0.026 & 0.144 \\
\hline ITGB3 & 0.711 & $0.445-1.135$ & 0.151 & 0.637 & $E G F R$ & 1.768 & $1.249-2.501$ & 0.001 & 0.006 & VCAMI & 0.651 & $0.44-0.962$ & 0.030 & 0.152 \\
\hline$P L A 2 G 2 A$ & 0.674 & $0.381-1.191$ & 0.171 & 0.637 & SPARC & 1.753 & $1.243-2.472$ & 0.001 & 0.006 & $A B C C I$ & 0.658 & $0.44-0.984$ & 0.040 & 0.183 \\
\hline$M G M T$ & 0.720 & $0.446-1.161$ & 0.176 & 0.637 & $D P Y D$ & 1.706 & $1.206-2.414$ & 0.002 & 0.010 & $P L A 2 G 2 A$ & 0.643 & $0.414-0.998$ & 0.047 & 0.183 \\
\hline REG4 & 0.721 & $0.446-1.165$ & 0.180 & 0.637 & MAPT & 1.804 & $1.214-2.681$ & 0.003 & 0.012 & $A B C B I$ & 0.659 & $0.435-0.998$ & 0.047 & 0.183 \\
\hline$C A V I$ & 0.728 & $0.456-1.163$ & 0.182 & 0.637 & LDHA & 0.626 & $0.445-0.881$ & 0.007 & 0.024 & $I G F I R$ & 1.466 & $0.996-2.158$ & 0.051 & 0.183 \\
\hline EZH2 & 1.395 & $0.854-2.278$ & 0.182 & 0.637 & ESRI & 1.626 & $1.139-2.319$ & 0.007 & 0.024 & $P L A U$ & 0.681 & $0.459-1.008$ & 0.053 & 0.183 \\
\hline$L R P 5$ & 1.362 & $0.850-2.183$ & 0.197 & 0.641 & $T O P 1$ & 0.645 & $0.458-0.908$ & 0.011 & 0.036 & $F P G S$ & 1.460 & $0.989-2.158$ & 0.056 & 0.183 \\
\hline VCAMI & 0.741 & $0.465-1.181$ & 0.206 & 0.641 & $B C L 2$ & 1.606 & $1.108-2.329$ & 0.012 & 0.036 & $M U C 2$ & 0.661 & $0.427-1.024$ & 0.062 & 0.192 \\
\hline GZMA & 0.742 & $0.460-1.198$ & 0.221 & 0.651 & $A B C B 1$ & 1.526 & $1.069-2.178$ & 0.019 & 0.056 & $B C L 2$ & 0.669 & $0.43-1.04$ & 0.072 & 0.206 \\
\hline$A B C C 1$ & 0.776 & $0.484-1.247$ & 0.293 & 0.776 & $R R M 2$ & 0.680 & $0.483-0.957$ & 0.026 & 0.073 & $R E G 4$ & 0.696 & $0.466-1.04$ & 0.076 & 0.206 \\
\hline$B C L 2$ & 0.768 & $0.451-1.308$ & 0.330 & 0.776 & $V C A M I$ & 1.453 & $1.032-2.045$ & 0.031 & 0.083 & $R U N X 3$ & 0.706 & $0.478-1.041$ & 0.077 & 0.206 \\
\hline$A P C$ & 0.797 & $0.500-1.268$ & 0.337 & 0.776 & $E R C C l$ & 1.401 & $1.001-1.961$ & 0.049 & 0.124 & $T Y M P$ & 0.735 & $0.498-1.085$ & 0.120 & 0.294 \\
\hline$H D A C l$ & 1.246 & $0.781-1.988$ & 0.356 & 0.776 & $T G F A$ & 1.405 & $0.993-1.988$ & 0.054 & 0.129 & PECAMI & 0.737 & $0.5-1.086$ & 0.121 & 0.294 \\
\hline TOPI & 1.245 & $0.779-1.991$ & 0.358 & 0.776 & $E 2 F 1$ & 0.704 & $0.491-1.010$ & 0.055 & 0.129 & $C A V 1$ & 0.763 & $0.518-1.122$ & 0.168 & 0.392 \\
\hline$L D H A$ & 1.220 & $0.766-1.942$ & 0.402 & 0.776 & $D A P K 1$ & 1.377 & $0.974-1.947$ & 0.069 & 0.148 & $A N G P T 2$ & 1.296 & $0.87-1.93$ & 0.200 & 0.449 \\
\hline$E R C C l$ & 0.822 & $0.516-1.308$ & 0.406 & 0.776 & $A R E G$ & 0.734 & $0.525-1.026$ & 0.069 & 0.148 & $R R M I$ & 1.263 & $0.856-1.863$ & 0.238 & 0.509 \\
\hline TGFA & 1.220 & $0.755-1.972$ & 0.416 & 0.776 & UMPS & 0.730 & $0.515-1.035$ & 0.076 & 0.148 & HPSE & 0.795 & $0.539-1.172$ & 0.246 & 0.509 \\
\hline SPARC & 0.829 & $0.521-1.320$ & 0.429 & 0.776 & $M U C 2$ & 0.718 & $0.497-1.037$ & 0.076 & 0.148 & $A P C$ & 0.819 & $0.556-1.204$ & 0.309 & 0.597 \\
\hline GADD $45 A$ & 1.232 & $0.717-2.115$ & 0.450 & 0.776 & GADD $45 A$ & 1.407 & $0.962-2.058$ & 0.077 & 0.148 & $M A P T$ & 1.257 & $0.807-1.958$ & 0.309 & 0.597 \\
\hline EREG & 1.229 & $0.711-2.124$ & 0.460 & 0.776 & $V E G F A$ & 0.743 & $0.531-1.042$ & 0.084 & 0.156 & $D P Y D$ & 0.823 & $0.558-1.214$ & 0.324 & 0.606 \\
\hline$A R E G$ & 0.846 & $0.531-1.348$ & 0.481 & 0.776 & $F A S$ & 1.345 & $0.952-1.899$ & 0.091 & 0.162 & DHFR & 0.834 & $0.568-1.227$ & 0.356 & 0.643 \\
\hline PTGS2 & 0.850 & $0.535-1.351$ & 0.491 & 0.776 & $A P C$ & 1.334 & $0.952-1.869$ & 0.093 & 0.162 & $B C L 2 L 11$ & 0.814 & $0.51-1.299$ & 0.387 & 0.666 \\
\hline$F P G S$ & 0.850 & $0.533-1.357$ & 0.495 & 0.776 & $P L A U$ & 1.315 & $0.939-1.842$ & 0.110 & 0.183 & MTHFR & 0.836 & $0.554-1.262$ & 0.393 & 0.666 \\
\hline$I G F 1 R$ & 1.168 & $0.735-1.854$ & 0.511 & 0.776 & PTGS2 & 1.321 & $0.934-1.867$ & 0.114 & 0.183 & $M L H I$ & 0.847 & $0.567-1.264$ & 0.414 & 0.683 \\
\hline$R U N X 3$ & 0.858 & $0.537-1.372$ & 0.522 & 0.776 & DUT & 0.763 & $0.545-1.069$ & 0.114 & 0.183 & PTEN & 0.859 & $0.583-1.265$ & 0.441 & 0.700 \\
\hline$M A P T$ & 0.845 & $0.499-1.431$ & 0.531 & 0.776 & REG4 & 1.308 & $0.929-1.843$ & 0.123 & 0.191 & $T Y M S$ & 1.160 & $0.789-1.705$ & 0.450 & 0.700 \\
\hline$V E G F A$ & 1.157 & $0.729-1.838$ & 0.536 & 0.776 & $B A X$ & 1.261 & $0.901-1.764$ & 0.175 & 0.265 & $H D A C l$ & 1.147 & $0.78-1.686$ & 0.484 & 0.713 \\
\hline MTHFR & 0.856 & $0.520-1.410$ & 0.541 & 0.776 & LRP5 & 1.232 & $0.880-1.725$ & 0.224 & 0.331 & $A R E G$ & 0.873 & $0.596-1.281$ & 0.488 & 0.713 \\
\hline$D P Y D$ & 0.866 & $0.545-1.376$ & 0.541 & 0.776 & MTHFR & 1.244 & $0.868-1.781$ & 0.233 & 0.335 & $E R E G$ & 0.855 & $0.543-1.345$ & 0.497 & 0.713 \\
\hline DHFR & 0.872 & $0.549-1.385$ & 0.561 & 0.786 & $P L A 2 G 2 A$ & 0.790 & $0.520-1.199$ & 0.267 & 0.374 & TOPI & 1.126 & $0.766-1.656$ & 0.545 & 0.762 \\
\hline$U M P S$ & 1.140 & $0.710-1.829$ & 0.587 & 0.795 & $H D A C l$ & 0.847 & $0.607-1.183$ & 0.330 & 0.451 & $R R M 2$ & 1.122 & $0.763-1.649$ & 0.558 & 0.762 \\
\hline$E 2 F 1$ & 1.132 & $0.708-1.810$ & 0.605 & 0.795 & PTEN & 0.851 & $0.609-1.191$ & 0.347 & 0.462 & $T G F A$ & 1.114 & $0.748-1.659$ & 0.596 & 0.794 \\
\hline$R R M 2$ & 1.124 & $0.703-1.797$ & 0.625 & 0.795 & $E R B B 2$ & 1.122 & $0.804-1.564$ & 0.500 & 0.651 & SPARC & 0.907 & $0.618-1.331$ & 0.617 & 0.804 \\
\hline DUT & 0.891 & $0.561-1.415$ & 0.625 & 0.795 & $E R E G$ & 0.877 & $0.589-1.307$ & 0.519 & 0.661 & $E R C C l$ & 1.087 & $0.741-1.594$ & 0.669 & 0.852 \\
\hline TYMS & 1.112 & $0.700-1.766$ & 0.652 & 0.812 & DHFR & 1.108 & $0.792-1.550$ & 0.547 & 0.681 & $D A P K l$ & 0.945 & $0.638-1.399$ & 0.778 & 0.957 \\
\hline$R R M I$ & 0.912 & $0.571-1.459$ & 0.701 & 0.853 & $M G M T$ & 1.088 & $0.773-1.531$ & 0.629 & 0.766 & $I T G B 3$ & 0.956 & $0.651-1.405$ & 0.819 & 0.957 \\
\hline$D A P K I$ & 1.086 & $0.675-1.748$ & 0.733 & 0.860 & $U P P I$ & 0.930 & $0.664-1.303$ & 0.673 & 0.801 & $L D H A$ & 1.034 & $0.703-1.522$ & 0.865 & 0.957 \\
\hline$M U C 2$ & 0.925 & $0.560-1.528$ & 0.761 & 0.860 & $R R M I$ & 0.939 & $0.670-1.314$ & 0.712 & 0.813 & $D U T$ & 1.033 & $0.703-1.518$ & 0.867 & 0.957 \\
\hline BCL2L11 & 0.917 & $0.521-1.616$ & 0.765 & 0.860 & MLHI & 1.065 & $0.751-1.508$ & 0.724 & 0.813 & $G A D D 45 A$ & 0.966 & $0.62-1.506$ & 0.879 & 0.957 \\
\hline
\end{tabular}




\begin{tabular}{|c|c|c|c|c|c|c|c|c|c|c|c|c|c|c|}
\hline$P L A U$ & 0.933 & $0.588-1.482$ & 0.769 & 0.860 & HPSE & 0.941 & $0.670-1.322$ & 0.726 & 0.813 & $E Z H 2$ & 0.970 & $0.653-1.442$ & 0.882 & 0.957 \\
\hline$A N G P T 2$ & 0.933 & $0.571-1.525$ & 0.783 & 0.860 & $A N G P T 2$ & 1.056 & $0.746-1.495$ & 0.760 & 0.835 & $M G M T$ & 0.973 & $0.661-1.431$ & 0.888 & 0.957 \\
\hline EGFR & 1.050 & $0.661-1.666$ & 0.837 & 0.884 & $R U N X 3$ & 0.970 & $0.692-1.359$ & 0.860 & 0.926 & $E 2 F I$ & 1.028 & $0.693-1.525$ & 0.889 & 0.957 \\
\hline HPSE & 1.044 & $0.658-1.657$ & 0.855 & 0.884 & $F P G S$ & 0.980 & $0.70-1.372$ & 0.908 & 0.960 & $B A X$ & 0.977 & $0.667-1.433$ & 0.906 & 0.957 \\
\hline$M L H I$ & 0.959 & $0.588-1.566$ & 0.867 & 0.884 & TYMP & 1.009 & $0.721-1.41$ & 0.960 & 0.989 & THBSI & 1.017 & $0.694-1.491$ & 0.931 & 0.962 \\
\hline THBSI & 1.040 & $0.655-1.651$ & 0.868 & 0.884 & GZMA & 1.005 & $0.708-1.428$ & 0.976 & 0.989 & $U P P 1$ & 0.987 & $0.672-1.449$ & 0.945 & 0.962 \\
\hline PTEN & 1.002 & $0.631-1.591$ & 0.993 & 0.993 & $B C L 2 L 11$ & 0.997 & $0.632-1.572$ & 0.989 & 0.989 & UMPS & 1.002 & $0.681-1.475$ & 0.992 & 0.992 \\
\hline
\end{tabular}

\section{Predictive value of biomarker analysis}

Results of the multivariate analysis of $G G H$, $P E C A M 1$, and TOP $2 A$ expression in association with the pattern of recurrence are shown in Supplementary Tables 3,4 , and 5 , respectively. These factors were found to be independent according to a logistic regression model, with hazard ratios that were comparable to those of tumor histological type.

\section{Gene expression correlations}

There was a statistically significant correlation between the mRNA expression levels of GGH, TOP2A, and PECAM1.TOP2A level was positively correlated with that of $G G H$ (Spearman's rank correlation coefficient: $\mathrm{r}$ $=0.57, P<0.0001$ ), while PECAM1 was negatively correlated with TOP2A $(\mathrm{r}=-0.25, P<0.0001)$ and $G G H$ $(\mathrm{r}=-0.34, P<0.0001)$. The correlation coefficient matrix of screened genes is shown in Supplementary Table 6.

\section{GGH, TOP2A, and PECAM1 gene expression according to tumor histology}

PECAM1 mRNA levels were higher in diffuse-type than in intestinal-type gastric cancer, whereas the opposite was true for TOP $2 A$ and $G G H$ expression $(P<0.0001)$.

\section{Subgroup analyses according to the disease stage}

We performed subgroup analyses according to the disease stage (II or III). The results are shown in Supplementary Table 7. In the disease stage subgroup analyses, none of the genes were correlated to lymph node recurrence. PECAM1 showed a significant correlation with peritoneal recurrence in both stage II (FDR $P=0.016$ ) and III patients (FDR $P=0.022$ ). In stage II patients, ATP binding cassette subfamily $\mathrm{C}$ member 1 and ERCC excision repair 1 , endonuclease non-catalytic subunit showed a stronger correlation with peritoneal recurrence than PECAM1 with FDR P values equal to 0.002 and 0.007 , respectively. TOP $2 A$ expression correlated significantly with hematogenous recurrence in stage III patients but not in stage II patients. The other genes were not found to be correlated to hematogenous recurrence more strongly than TOP $2 A$ in the subgroup analyses according to tumor stage.

\section{Subgroup analyses according to the intervention}

We performed subgroup analyses according to the intervention (surgery alone or S-1). The results are shown in Table S8. In the intervention subgroup analyses, none of the genes were correlated to lymph node recurrence. PECAM1 showed a significant correlation to peritoneal recurrence in both surgery alone (FDR $P=0.005)$ and S-1 treated patients (FDR $P=0.031$ ). In the surgery alone group, enhancer of zeste 2 polycomb repressive complex 2 subunit showed a stronger correlation to peritoneal recurrence than PECAM1 with FDR P value 0.004. TOP2A expression correlated significantly with hematogenous recurrence in the surgery alone group but not in S-1 treated patients. Other genes were not found to be correlated to hematogenous recurrence more strongly than TOP $2 A$ in the surgery alone group.

No statistically significant interaction for sitespecific recurrence was observed between 56 gene expressions and the treatment type (S-1 vs. Surgery alone) (Supplementary Table 9).

\section{DISCUSSION}

In the present study, the relationship between the expression levels of 63 preselected genes and specific recurrence sites in patients with stage II/III gastric cancer was retrospectively analyzed. We found that TOP 2A, GGH, and PECAM1 levels were associated with hematogenous, lymph node, and peritoneal recurrence, respectively. In contrast, there were no genes that could explain local recurrence given the limited number of events.

TOP $2 A$ expression was highly correlated with hematogenous recurrence. High TOP2A expression increases the risk of hematogenous recurrence $(\mathrm{HR}=2.353)$, but it is also a good prognostic factor for peritoneum recurrences $(\mathrm{HR}=0.489)$. Topoisomerase II, which is involved in DNA synthesis and cell proliferation, is the target of antitumor drugs such as anthracyclines. There are a few reports directly linking TOP2A gene expression level and tumor hematogenous recurrence. However, topoisomerase II inhibitors such as razoxane inhibit the metastatic spread of tumors in experimental 
animals and possess antiangiogenic activity [13]. Correlation analysis revealed that the Pearson correlation coefficient between $T O P 2 A$ and receptor tyrosine kinase $E r b-b 2$ (ERBB2) in 832 samples was 0.403 (CI: 0.3439 $0.45 ; P<0001)$. ERBB2 was the gene that was most highly correlated with hematogenous recurrence after TOP $2 A(\mathrm{HR}=2.23$; 95\% CI: $1.48-3.34 ; P<0.0001$; FDR $=0.002)$. Co-amplification of ERBB2 and TOP $2 A$ has been reported in breast cancer cases, which has been attributed to their proximity to chromosome 17 (17q12 and 17q21.322 , respectively) [14]. Thus, the same may be said of coamplification of $E R B B 2$ and TOP $2 A$ in gastric cancer.

$G G H$ showed the strongest correlation among the screened genes with lymph node recurrence; FDR for $G G H$ was 0.345 . However, tumor-stage and intervention subgroup analyses showed that $G G H$ did not have any statistically significant correlation with lymph node recurrence. False positivity remains a concern for this gene. $G G H$ encodes an enzyme that catalyzes the hydrolysis of (anti) folylpoly-gamma-glutamates by the removal of gamma-linked polyglutamates and regulates intracellular folate concentrations. $G G H$ is reportedly a prognostic factor for tumors. In an animal model of lung cancer using tail vain injection of A549 cells in SCID mice, folate modulating metastatic potential was confirmed. A folate-rich diet increased lung colonization, increased distant metastasis to lymph nodes, and decreased overall survival $[15,16]$. However, there have been no studies to date on the association between $G G H$ and gastric cancer. $G G H$ upregulation reduces folic acid levels in tumor cells; thus, leucovorin combination therapy may reduce the risk of lymph node metastasis. An open-label, randomized, multi-center phase III study of TAS-118 (a tegafur-gimeracil-oteracil potassium-leucovorin calcium oral formulation) plus oxaliplatin vs. S-1 plus cisplatin as a first-line therapy in patients with advanced gastric cancer is currently recruiting participants [17].

PECAM1, which was significantly correlated with peritoneal recurrence, encodes a protein involved in several processes relevant to the growth and spread of primary tumors, including angiogenesis, vascular permeability, and leukocyte trafficking out of the
A
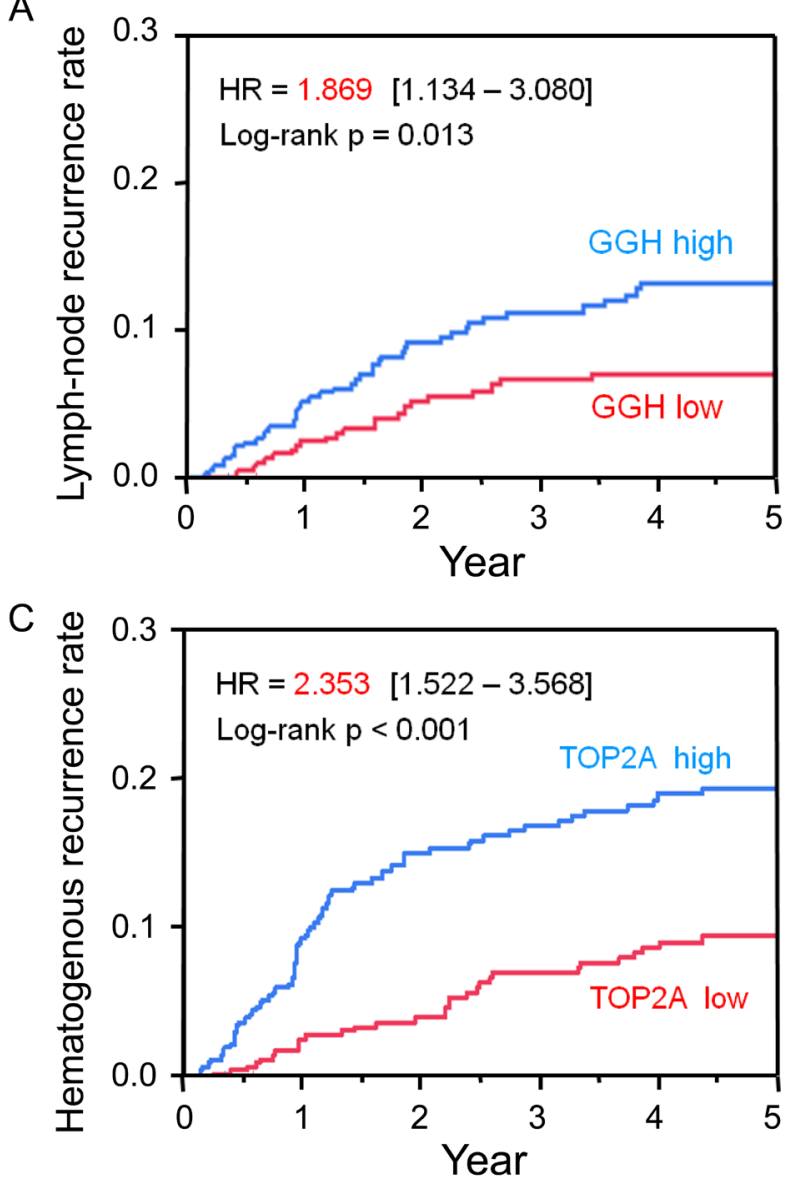

B

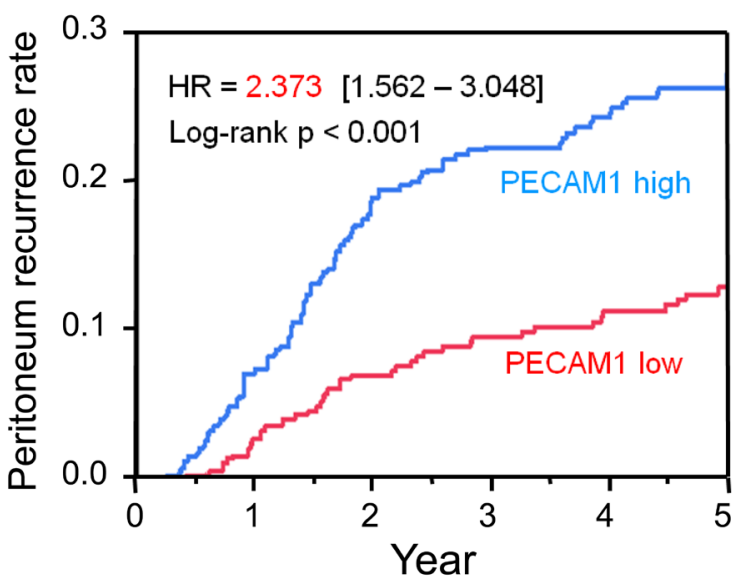

Figure 2: Risk factors for recurrence at each site. A. Five-year cumulative lymph node recurrence rate for patients with $G G H$ high (blue) and $G G H$-low (red) tumors. B. Five-year cumulative peritoneum recurrence rate for patients with $P E C A M 1$-high (blue) and PECAM1-low (red) tumors. C. Five-year cumulative hematogenous recurrence rate for patients with TOP $2 A$-high (blue) and TOP $2 A$-low (red) tumors. 
circulation [18]. PECAM1 promotes neovascularization in the peritoneum, cell migration, and metastatic progression. PECAM1 inhibition has been suggested as a therapeutic approach that targets the tumor microenvironment to suppress the end stages of metastatic progression, which has until now been a refractory clinical entity [19].

This study is the first to describe key genes that are significantly associated with recurrence at specific sites in patients with gastric cancer who have undergone 5-fluorouracil-based adjuvant chemotherapy. The understanding of molecular profiles associated with postoperative relapse risk may contribute to the development of proper preoperative monitoring procedures that make it possible to detect cancer recurrence earlier and more precisely based on gene expression profiling of resected tumor samples. Nevertheless, there were several limitations to the study. Firstly, it is a retrospectiveprospective, designed, single exploratory biomarker study following randomized phase III clinical trials. The prognostic significance of the identified genes must be validated in a biomarker-based multi-institutional study of a comparable number of patients. Secondly, we screened a relatively small number of genes that were preselected based on published reports since we were unable to establish a reliable genome-wide profiling procedure on partly damaged RNA extracted from FFPE tissue samples. Thirdly, we could not evaluate protein levels by clinically feasible approaches such as immunohistochemistry because of the unavailability of specimens to validate candidate genes. Despite these limitations, our findings can contribute to the development of targeted/combination therapies for patients with gastric cancer who are at a high risk of relapse.

\section{MATERIALS AND METHODS}

\section{Study population and design}

Tumor tissue samples were collected from patients enrolled in the ACTS-GC study; the inclusion criteria and treatment protocol have been previously described [2]. The protocol used in the present study was approved by the ethics committee of the Japanese Gastric Cancer Association and the institutional review board of each participating hospital, and was in compliance with the Reporting Recommendations for Tumor Marker Prognostic Studies guidelines [20].

\section{Quantitative real-time PCR}

Representative hematoxylin and eosin-stained slides from formalin-fixed, paraffin-embedded (FFPE) specimens were reviewed by a pathologist to estimate the tumor load. Sections (10 $\mu \mathrm{m}$ in thickness) were stained with nuclear Fast Red (Sigma-Aldrich, St. Louis, MO, USA) for manual microdissection of tumor tissue, which was carried out using a scalpel as previously described [8].

RNA was isolated from tumor tissue and cDNA was prepared as previously reported [21], with a minor modification to the extraction step for which we used RNeasy Mini Elute spin columns (Qiagen, Valencia, CA, USA). Expression levels of 63 genes were determined by qRT-PCR using a TaqMan array card (Life Technologies, Carlsbad, CA, USA) after TaqMan assay-based preamplification. Briefly, cDNA $(2.5 \mu \mathrm{l})$ was pre-amplified using the TaqMan PreAmp Master Mix $(2 \times)$ and a pool of TaqMan Gene Expression Assays (0.2×) in a 10- $\mu$ l PCR reaction. The cycling conditions were $95^{\circ} \mathrm{C}$ for $10 \mathrm{~min}$, followed by 14 cycles of $95^{\circ} \mathrm{C}$ for $15 \mathrm{~s}$ and $60^{\circ} \mathrm{C}$ for $4 \mathrm{~min}$. The amplified cDNA sample was diluted 20 fold in TrisEDTA buffer, and $25 \mu 1$ were added to $25 \mu 1$ of RNase-free water and $50 \mu \mathrm{l}$ of $2 \times$ TaqMan Gene Expression Master Mix. The mixture was transferred to a loading port on the TaqMan low-density array (LDA), which was centrifuged twice and sealed. PCR amplification was carried out on a Prism 7900HT Sequence Detection System (Applied Biosystems, Foster City, CA, USA) under the following conditions: $50^{\circ} \mathrm{C}$ for $2 \mathrm{~min}$ and $94.5^{\circ} \mathrm{C}$ for $10 \mathrm{~min}$, followed by 40 cycles of $97^{\circ} \mathrm{C}$ for $30 \mathrm{~s}$ and $59.7^{\circ} \mathrm{C}$ for $1 \mathrm{~min}$. The LDA included $\beta$-actin, glyceraldehyde 3-phosphate dehydrogenase, and ribosomal protein lateral stalk subunit $P 0$ as reference genes [22, 23]. Assay IDs used in the LDA are shown in Supplementary Table 1 hyperlinked to the DAVID website $[24,25]$. The LDA includes the assays for genes encoding key molecules for growth factor signaling pathways, apoptotic signaling pathways, and DNA repair mechanisms, as well as 5-FU metabolism related genes $[26,27]$. The cycle threshold value - which is inversely proportional to the amount of cDNA - was calculated. Relative mRNA levels are expressed as the ratio between the gene of interest and the geometric mean of the reference genes, providing a baseline measurement for the amount of mRNA isolated from each specimen. The expression level of each gene was categorized as low or high relative to the 50 th percentile (median).

\section{Data processing and statistical analysis}

The geNORM algorithm (MS-Excel add-on macro program; Microsoft, Redmond, WA, USA) was used to determine the stability of the reference genes as previously described [22]. The program calculated the gene stability, $\mathrm{M}$, by determining the average pairwise variation between a particular reference gene and all other control genes. A normalization factor was calculated for genes with $\mathrm{M}<$ 1.5 based on the geometric mean of expression levels of selected genes. For quality control, only target genes for which data were obtained from $>60 \%$ of samples were used while the remainder were excluded from further analysis. 
Categorical data were analyzed with the $\chi^{2}$ test. The Wilcoxon or Kruskal-Wallis test was used to assess correlations between groups. Survival curves were generated with the Kaplan-Meier product-limit method, and differences between them were evaluated with the log-rank test. Uni- and multivariate survival analyses were performed with a Cox proportional hazards model. Results were considered statistically significant at $P<$ 0.05. All statistical analyses were carried out using SAS package v.9.1, JMP v.8.01 (SAS Institute, Cary, NC, USA), and MS-Excel (add-on macro program) software. The Benjamini and Hochberg false discovery rate (FDR) controlling procedure was used for multiple comparisons. Correlations between gene expression and prognosis were considered statistically significant at FDR $P<0.10$.

\section{Statement of translational relevance}

This study identifies genes whose expression strongly correlates with specific gastric cancer relapse sites. Resection, coupled with adjuvant therapy, can drastically increase survival in gastric cancer patients. However, some cancers recur in the form of blood-borne metastasis or peritoneal dissemination. It is not known why and how this occurs, in part due to the high variability among patients exhibiting recurrence. We carried out a retrospective analysis of surgical samples to investigate whether there is a correlation between relapse site and the expression of specific genes, and found that high expression of topoisomerase II alpha, gamma-glutamyl hydrolase, and platelet/endothelial cell adhesion molecule 1 correlates with high risk of hematogenous, lymph node, and peritoneal recurrence, respectively, in patients with stage II/III gastric cancer.

\section{ACKNOWLEDGMENTS}

This manuscript is dedicated to the memory of Prof. Tetsuro Kubota, who made a significant contribution to the conception and design of this study. The authors thank the patients, their families, and investigators from the 65 participating institutions for their cooperation.

\section{CONFLICTS OF INTEREST}

The results of this study were presented, in part, at the 2013 American Society of Clinical Oncology annual meeting held on May 30-June 3 in Chicago, IL, USA.

\section{FINANCIAL SUPPORT}

This study was funded by Taiho Pharmaceutical Co., Tokyo, Japan.

\section{REFERENCES}

1. Shirasaka T, Shimamato Y, Ohshimo H, Yamaguchi M, Kato T, Yonekura K, Fukushima M. Development of a novel form of an oral 5-fluorouracil derivative (S-1) directed to the potentiation of the tumor selective cytotoxicity of 5-fluorouracil by two biochemical modulators. Anticancer Drugs. 1996; 7: 548-57.

2. Sakuramoto S, Sasako M, Yamaguchi T, Kinoshita T, Fujii M, Nashimoto A, Furukawa H, Nakajima T, Ohashi Y, Imamura H, Higashino M, Yamamura Y, Kurita A, et al. Adjuvant chemotherapy for gastric cancer with S-1, an oral fluoropyrimidine. N Engl J Med. 2007; 357: 1810-20.

3. Sasako M, Sakuramoto S, Katai H, Kinoshita T, Furukawa H, Yamaguchi T, Nashimoto A, Fujii M, Nakajima T, Ohashi Y. Five-year outcomes of a randomized phase III trial comparing adjuvant chemotherapy with S-1 versus surgery alone in stage II or III gastric cancer. J Clin Oncol. 2011; 29: 4387-93.

4. Mastoraki A, Papanikolaou IS, Sakorafas G, Safioleas M. Facing the challenge of managing linitis plastica - review of the literature. Hepatogastroenterology. 2009; 56: 177378.

5. Tan IB, Ivanova $\mathrm{T}$, Lim KH, Ong CW, Deng N, Lee J, Tan SH, Wu J, Lee MH, Ooi CH, Rha SY, Wong WK, Boussioutas A, et al. Intrinsic subtypes of gastric cancer, based on gene expression pattern, predict survival and respond differently to chemotherapy. Gastroenterology. 2011; 141: 476-85.

6. Wittner BS, Sgroi DC, Ryan PD, Bruinsma TJ, Glas AM, Male A, Dahiya S, Habin K, Bernards R, Haber DA, Van't Veer LJ, Ramaswamy S. Analysis of the MammaPrint breast cancer assay in a predominantly postmenopausal cohort. Clin Cancer Res. 2008; 14: 2988-93.

7. Salazar R, Roepman P, Capella G, Moreno V, Simon I, Dreezen C, Lopez-Doriga A, Santos C, Marijnen C, Westerga J, Bruin S, Kerr D, Kuppen P, et al. Gene expression signature to improve prognosis prediction of stage II and III colorectal cancer. J Clin Oncol. 2011; 29: $17-24$.

8. Ichikawa W. Prediction of clinical outcome of fluoropyrimidine-based chemotherapy for gastric cancer patients, in terms of the 5-fluorouracil metabolic pathway. Gastric Cancer. 2006; 9: 145-55.

9. Watanabe $\mathrm{T}$, Kobunai $\mathrm{T}$, Yamamoto Y, Kanazawa T, Konishi T, Tanaka T, Matsuda K, Ishihara S, Nozawa $\mathrm{K}$, Eshima K, Muto T, Nagawa $H$. Prediction of liver metastasis after colorectal cancer using reverse transcription-polymerase chain reaction analysis of 10 genes. Eur J Cancer. 2010; 46: 2119-26.

10. Takeno A, Takemasa I, Seno S, Yamasaki M, Motoori M, Miyata H, Nakajima K, Takiguchi S, Fujiwara Y, Nishida T, Okayama T, Matsubara K, Takenaka Y, et al. Gene expression profile prospectively predicts peritoneal relapse after curative surgery of gastric cancer. Ann Surg Oncol. 
2010; 17: 1033-42.

11. Lee J, Sohn I, Do IG, Kim KM, Park SH, Park JO, Park YS, Lim HY, Sohn TS, Bae JM, Choi MG, Lim DH, Min BH, et al. Nanostring-based multigene assay to predict recurrence for gastric cancer patients after surgery. PLoS ONE. 2014; 9: e90133.

12. Terashima M, Kitada K, Ochiai A, Ichikawa W, Kurahashi I, Sakuramoto S, Katai H, Sano T, Imamura H, Sasako $\mathrm{M}$; ACTS-GC Group. Impact of expression of human epidermal growth factor receptors EGFR and ERBB2 on survival in stage II/III gastric cancer. Clin Cancer Res. 2012; 18: 5992-6000.

13. Braybrooke JP, O'Byrne KJ, Propper DJ, Blann A, Saunders M, Dobbs N, Han C, Woodhull J, Mitchell K, Crew J, Smith K, Stephens R, Ganesan TS, et al. A phase II study of razoxane, an antiangiogenic topoisomerase II inhibitor, in renal cell cancer with assessment of potential surrogate markers of angiogenesis. Clin Cancer Res. 2000; 6: 4697-704.

14. Varga Z, Tubbs RR, Wang X, Sun Y, Noske A, Kradolfer D. Co-amplification of the HER2 gene and chromosome 17 centromere: a potential diagnostic pitfall in HER2 testing in breast cancer. Breast Cancer Res Treat. 2012; 132: 925-35.

15. Oleinik NV, Helke KL, Kistner-Grifin E, Krupenko NI, Krupenko SA. Rho GTPases RhoA and Racl mediate effects of dietary folate on metastatic potential of A549 cancer cells through the control of cofilin phosphorylation. J Biol Chem. 2014; 289: 26383-94.

16. Shubbar E, Helou K, Kovács A, Nemes S, Hajizadeh S, Enerbäck C, Einbeigi Z. High levels of $\gamma$-glutamyl hydrolase $(\mathrm{GGH})$ are associated with poor prognosis and unfavorable clinical outcomes in invasive breast cancer. BMC Cancer. 2013; 13: 47.

17. ClinicalTrials.gov [https:/clinicaltrials.gov/ct2/show/ NCT02322593]

18. Piali L, Hammel P, Uherek C, Bachmann F, Gisler RH, Dunon D, Imhof BA. CD31/PECAM-1 is a ligand for $\alpha v \beta 3$ integrin involved in adhesion of leukocytes to endothelium. J Cell Biol. 1995; 130: 451-60.

19. DeLisser H, Liu Y, Desprez PY, Thor A, Briasouli P,
Handumrongkul C, Wilfong J, Yount G, Nosrati M, Fong S, Shtivelman E, Fehrenbach M, Cao G, et al. Vascular endothelial platelet endothelial cell adhesion molecule 1 (PECAM-1) regulates advanced metastatic progression. Proc Natl Acad Sci USA. 2010; 107: 18616-21.

20. McShane LM, Altman DG, Sauerbrei W, Taube SE, Gion M, Clark GM; Statistics Subcommittee of the NCIEORTC Working Group on Cancer Diagnostics. Reporting recommendations for tumor marker prognostic studies (REMARK). J Natl Cancer Inst. 2005; 97: 1180-84.

21. Ceppi P, Volante M, Novello S, Rapa I, Danenberg KD, Danenberg PV. ERCC1 and RRM1 gene expressions but not EGFR are predictive of shorter survival in advanced non-small-cell lung cancer treated with cisplatin and gemcitabine. Ann Oncol. 2006; 17: 1818-25.

22. Pérez S, Royo LJ, Astudillo A, Escudero D, Álvarez F, Rodríguez A, Gómez E, Otero J. Identifying the most suitable endogenous control for determining gene expression in hearts from organ donors. BMC Mol Biol. 2007; 8: 114.

23. Vandesompele J, De Preter K, Pattyn F, Poppe B, Van Roy N, De Paepe A, Speleman F. Accurate normalization of real-time quantitative RT-PCR data by geometric averaging of multiple internal control genes. Genome Biol. 2002; 3: RESEARCH0034.

24. Huang DW, Sherman BT, Lempicki RA. Systematic and integrative analysis of large gene lists using DAVID Bioinformatics Resources. Nature Protoc. 2009; 4: 44-57.

25. Huang DW, Sherman BT, Lempicki RA. Bioinformatics enrichment tools: paths toward the comprehensive functional analysis of large gene lists. Nucleic Acids Res. 2009; 37: 1-13.

26. Fareed KR, Kaye P, Soomro IN, Ilyas M, Martin S, Parsons SL, Madhusudan S. Biomarkers of response to therapy in oesophago-gastric cancer. Gut. 2009; 58: 127-43.

27. Ichikawa W. Prediction of clinical outcome of fluoropyrimidine-based chemotherapy for gastric cancer patients, in terms of the 5-fluorouracil metabolic pathway. Gastric Cancer. 2006; 9: 145-55. 